Journal of Scientific Perspectives

Volume 3, Issue 1, Year 2019, pp. 21-28

E - ISSN: 2587-3008

URL: http://ratingacademy.com.tr/ojs/index.php/jsp

DOİ: $10.26900 /$ jsp.3.003

Research Article

\title{
SOME HIGHER ORDER DIFFERENCE DOUBLE SEQUENCE SPACES DEFINED BY AN ORLICZ FUNCTION
}

\author{
Bipul SARMA * \\ *MC College (Gauhati University), Barpeta, Assam, INDIA, E-mail: drbsar@yahoo.co.in \\ ORCID ID: https://orcid.org/0000-0003-4446-6710
}

Received: 9 December 2018; Accepted: 9 January 2019

\section{ABSTRACT}

In this article we introduce some kth order difference operator on some double sequences operated by an Orlicz function. We introduce some sequence spaces and study different properties of these spaces like completeness, solidity, symmetricity etc. We establish some inclusion results among them.

Keywords: Orlicz function, difference space, completeness, solid space, symmetric space etc..

2010 AMS Subject Classification: 40A05; 40B05; 46E30

\section{INTRODUCTION}

Throughout, a double sequence is denoted by $A=\left\langle a_{i j}\right\rangle$. A double sequence is a double infinite array of elements $a_{i j} \in R$ for all $i, j \in N$ and ${ }_{2} w$ will denote the class of all double sequences.

The initial works on double sequences is found in Bromwich [2]. Later on it was studied by Hardy [6], Moricz [12], Moricz and Rhoades [13], Tripathy [16], Tripathy and Sarma [17], Tripathy, Choudhury and Sarma [18], Basarir and Sonalcan [1] and many others. Hardy [6] introduced the notion of regular convergence for double sequences.

The concept of paranormed sequences was studied by Nakano [14] and Simmons [15] at the initial stage. Later on it was studied by many others.

The notion of difference sequence spaces (for single sequences) was introduced by

Kizmaz [8] as follows:

$$
Z(\Delta)=\left\{\left(x_{k}\right) \in w:\left(\Delta x_{k}\right) \in Z\right\}
$$

for $Z=c, c_{0}$ and $1_{\infty}$, where $\Delta x_{k}=x_{k}-x_{k+1}$ for all $k \in N$. 
The above spaces are Banach spaces, normed by

$$
\left\|\left(x_{k}\right)\right\|=\left|x_{1}\right|+\sup _{k \geq 1}\left|\Delta x_{k}\right|
$$

The notion was further investigated by many workers on sequence spaces.

\section{DEFINITIONS AND PRELIMINARIES}

An Orlicz function $M$ is a mapping $M:[0, \infty) \rightarrow[0, \infty)$ such that it is continuous, nondecreasing and convex with $M(0)=0, M(x)>0$, for $x>0$ and $M(x) \rightarrow \infty$, as $x \rightarrow \infty$.

Lindenstrauss and Tzafriri [10] used the idea of Orlicz function to construct the sequence space

$$
1^{M}=\left\{\left(x_{k}\right): \sum_{k=1}^{\infty} M\left(\frac{\left|x_{k}\right|}{\rho}\right), \text { for some } \rho>0\right\},
$$

which is a Banach space normed by

$$
\left\|\left(x_{k}\right)\right\|=\inf \left\{\rho>0: \sum_{k=1}^{\infty} M\left(\frac{\left|x_{k}\right|}{\rho}\right) \leq 1\right\} .
$$

The space $\lambda^{M}$ is closely related to the space $\lambda^{p}$, which is an Orlicz sequence space with $M(x)=|x|^{p}$, for $1 \leq p<\infty$.

An Orlicz function $M$ is said to satisfy the $\Delta_{2}$-condition for all values of $u$, if there exists a constant $K>0$, such that $M(2 u) \leq K(M u), u \geq 0$.

Remark 1. Let $0<\lambda<1$, then $M(\lambda x) \leq \lambda M(x)$, for all $x \geq 0$.

Let $p=\left(p_{k}\right)$ be a positive sequence of real numbers. If $0<p_{k} \leq \sup p_{k}=H$ and $D=\max$ $\left(1,2^{H-1}\right)$, then for $a_{k}, b_{k} \in C$ for all $k \in N$, we have

$$
\left|a_{k}+b_{k}\right|^{p_{k}} \leq D\left\{\left|a_{k}\right|^{p_{k}}+\left|b_{k}\right|^{p_{k}}\right\} .
$$

Definition 2.1. A double sequence space $E$ is said to be solid if $\left\langle\alpha_{i j} a_{i j}\right\rangle \in E$ whenever $\left\langle a_{i j}\right\rangle \in E$ for all double sequences $\left\langle\alpha_{i j}\right\rangle$ of scalars with $\left|\alpha_{i j}\right| \leq 1$ for all $i, j \in N$.

Definition 2.2. Let $K=\left\{\left(n_{i}, k_{i}\right): i \in N ; n_{1}<n_{2}<n_{3}<\ldots\right.$ and $\left.k_{1}<k_{2}<k_{3}<\ldots\right\}$

$\subseteq N \times N$ and $E$ be a double sequence space. A $K$-step space of $E$ is a sequence space $\lambda_{K}^{E}=\left\{<a_{n_{i} k_{i}}>\in{ }_{2} w:<a_{n k}>\in E\right\}$.

A canonical pre-image of a sequence $\left\langle a_{n_{i} k_{i}}\right\rangle \in E$ is a sequence $\left\langle b_{n k}\right\rangle \in E$ defined as follows:

$$
b_{n k}= \begin{cases}a_{n k}, & \text { if }(n, k) \in K \\ 0, & \text { otherwise }\end{cases}
$$

A canonical pre-image of a step space $\lambda_{K}^{E}$ is a set of canonical pre-images of all elements in $\lambda_{K}^{E}$.

Definition 2.3. A double sequence space $E$ is said to be monotone if it contains the canonical pre-images of all its step spaces. 
Remark 2. From the above notions, it follows that "If a sequence space $E$ solid then $E$ is monotone".

Definition 2.4. A double sequence space $E$ is said to be symmetric if $\left\langle a_{i j}\right\rangle \in E$ implies $\left\langle a_{\pi(i) \pi(j)}\right\rangle \in E$, where $\pi$ is the permutation of $N$.

Let $M$ be an Orlicz function and $p=\left\langle p_{i j}\right\rangle$ be a double sequence of strictly positive real numbers. We introduce the following sequence spaces.

$$
\begin{aligned}
& { }_{2} W\left(M, \Delta^{k}, p\right)=\left\{<a_{i j}>\in_{2} w: \lim _{m, n} \frac{1}{m n} \sum_{i=1}^{m} \sum_{j=1}^{n}\left(M\left(\frac{\left|\Delta^{k} a_{i j}-L\right|}{\rho}\right)\right)^{p_{i j}}=0,\right. \\
& \text { for some } \rho>0 \text { and } L .\} \\
& { }_{2} W_{0}\left(M, \Delta^{k}, p\right)=\left\{<a_{i j}>\in{ }_{2} w: \lim _{m, n} \frac{1}{m n} \sum_{i=1}^{m} \sum_{j=1}^{n}\left(M\left(\frac{\left|\Delta^{k} a_{i j}\right|}{\rho}\right)\right)^{p_{i j}}=0 \text {, for some } \rho>0 .\right\} \\
& { }_{2} W_{\infty}\left(M, \Delta^{k}, p\right)=\left\{<a_{i j}>\in{ }_{2} w: \sup _{m, n} \frac{1}{m n} \sum_{i=1}^{m} \sum_{j=1}^{n}\left(M\left(\frac{\left|\Delta^{k} a_{i j}\right|}{\rho}\right)\right)^{p_{i j}}<\infty, \text { for some } \rho>0 .\right\}
\end{aligned}
$$

\section{RESULTS}

Theorem 3.1. The sequence spaces ${ }_{2} W\left(M, \Delta^{k}, p\right),{ }_{2} W_{0}\left(M, \Delta^{k}, p\right)$ and ${ }_{2} W_{\infty}\left(M, \Delta^{k}, p\right)$ are paranormed sequence spaces paranormed by

$$
\begin{aligned}
g\left(\left\langle a_{i j}\right\rangle\right)=\inf \left\{\rho^{\frac{p_{i j}}{J}}>0: \sup _{i, j}\left(M\left(\frac{\left|\Delta^{k} a_{i j}\right|}{\rho}\right)\right)\right. & \leq 1\}, \\
& \text { where } J=\max (1, H) .
\end{aligned}
$$

Proof. Clearly $g(0)=0, g\left(-\left\langle a_{i j}\right\rangle\right)=g\left(\left\langle a_{i j}\right\rangle\right)$. Let $\left\langle a_{i j}\right\rangle,\left\langle b_{i j}\right\rangle \in{ }_{2} W_{\infty}\left(M, \Delta^{k}, p\right)$. Then there exists some $\rho_{1}, \rho_{2}>0$ such that

$$
\begin{aligned}
& \sup _{i, j}\left(M\left(\frac{\left|\Delta^{k} a_{i j}\right|}{\rho_{1}}\right)\right) \leq 1 \\
& \text { and } \sup _{i, j}\left(M\left(\frac{\left|\Delta^{k} b_{i j}\right|}{\rho_{2}}\right)\right) \leq 1 .
\end{aligned}
$$

Let $\rho=\rho_{1}+\rho_{2}$. Then we have,

$\sup _{m, n}\left(M\left(\frac{\left|\Delta^{k} a_{i j}+\Delta^{k} b_{i j}\right|}{\rho}\right)\right) \leq \frac{\rho_{1}}{\rho_{1}+\rho_{2}} \sup _{i, j}\left(M\left(\frac{\left|\Delta^{k} a_{i j}\right|}{\rho_{2}}\right)\right)+\frac{\rho_{2}}{\rho_{1}+\rho_{2}} \sup _{i, j}\left(M\left(\frac{\left|\Delta^{k} b_{i j}\right|}{\rho_{2}}\right)\right) \leq 1$

Now 


$$
\begin{aligned}
& \mathrm{g}\left(<a_{i j}>+<b_{i j}>\right)=\inf \left\{\left(\rho_{1}+\rho_{2}\right)^{\frac{p_{i j}}{J}}>0: \sup _{i, j}\left(M\left(\frac{\left|\Delta^{k} a_{i j}+\Delta^{k} b_{i j}\right|}{\rho_{1}+\rho_{2}}\right)\right) \leq 1\right\} \\
& \leq \inf \left\{\rho_{1}^{\frac{p_{i j}}{J}}: \sup _{i, j}\left(M\left(\frac{\left|\Delta^{k} a_{i j}\right|}{\rho_{1}}\right)\right) \leq 1\right\}+\inf \left\{\rho_{2}^{\frac{p_{i j}}{J}}: \sup _{m, n}\left(M\left(\frac{\left|\Delta^{k} b_{i j}\right|}{\rho_{2}}\right)\right) \leq 1\right\} \\
& =g\left(<a_{i j}>\right)+g\left(<b_{i j}>\right)
\end{aligned}
$$

Let $\eta \in C$, then the continuity of the product follows from the following equality.

$$
\begin{aligned}
g\left(\eta<a_{i j}>\right) & =\inf \left\{\rho^{\frac{p_{i j}}{J}}: \sup _{i, j}\left(M\left(\frac{\left|\Delta^{k} \eta a_{i j}\right|}{\rho}\right)\right) \leq 1, \rho>0\right\} \\
& =\inf \left\{(|\eta| r)^{\frac{p_{i j}}{J}}: \sup _{m, n}\left(M\left(\frac{\left|\Delta^{k} a_{i j}\right|}{r}\right)\right) \leq 1, r>0\right\},
\end{aligned}
$$

where $\frac{1}{r}=\frac{|\eta|}{\rho}$.

Proposition 3.2. (i) ${ }_{2} W\left(M, \Delta^{k}, p\right) \subset{ }_{2} W_{\infty}\left(M, \Delta^{k}, p\right) \quad(i i){ }_{2} W_{0}\left(M, \Delta^{k}, p\right) \subset{ }_{2} W_{\infty}\left(M, \Delta^{k}\right.$, p). The inclusions are strict.

Theorem 3.3. If $\sup \frac{p_{i j}}{p_{u v}}<\infty$ for all $i \geq u, j \geq v$, then ${ }_{2} W\left(M, \Delta^{k-1}, p\right) \subset{ }_{2} W_{0}\left(M, \Delta^{k}\right.$, p). The inclusion is strict.

Proof. Let $<a_{i j}>\in{ }_{2} W\left(M, \Delta^{k-1}, p\right)$. Then

$$
\lim _{m, n} \frac{1}{m n} \sum_{i=1}^{m} \sum_{j=1}^{n}\left(M\left(\frac{\left|\Delta^{k-1} a_{i j}-L\right|}{\rho}\right)\right)^{p_{i j}}=0 \text {, for some } \rho>0 \text { and } L \text {. }
$$

Since $\sup \frac{p_{i j}}{p_{u v}}<\infty$ so there exists $K>0$ such that $p_{i j}<K$. $p_{u v}$ for all $i \geq u, j \geq v$.

Thus from (2) we have, $\lim _{m, n} \frac{1}{m n} \sum_{i=1}^{m} \sum_{j=1}^{n}\left(M\left(\frac{\left|\Delta^{k-1} a_{i j}-L\right|}{\rho}\right)\right)^{p_{i, j+1}}=0$,

$$
\begin{aligned}
& \lim _{m, n} \frac{1}{m n} \sum_{i=1}^{m} \sum_{j=1}^{n}\left(M\left(\frac{\left|\Delta^{k-1} a_{i j}-L\right|}{\rho}\right)\right)^{p_{i+1, j}}=0 \text { and } \\
& \lim _{m, n} \frac{1}{m n} \sum_{i=1}^{m} \sum_{j=1}^{n}\left(M\left(\frac{\left|\Delta^{k-1} a_{i j}-L\right|}{\rho}\right)\right)^{p_{i+1, j+1}}=0 .
\end{aligned}
$$

Now for $\left|\Delta^{k} a_{i j}\right|=\left|\Delta^{k-1}\left(a_{i j}-a_{i, j+1}-a_{i+1, j}+a_{i+1, j+1}\right)\right|$

$$
=\left|\Delta^{k-1} a_{i j}-\Delta^{k-1} a_{i, j+1}-\Delta^{k-1} a_{i+1, j}+\Delta^{k-1} a_{i+1, j+1}+L-L+L-L\right|
$$


we have,

$$
\begin{gathered}
\lim _{m, n} \frac{1}{m n} \sum_{i=1}^{m} \sum_{j=1}^{n}\left(M\left(\frac{\left|\Delta^{k} a_{i j}\right|}{\rho}\right)\right)^{p_{i j}} \\
\leq \lim _{m, n} \frac{1}{m n} \sum_{i=1}^{m} \sum_{j=1}^{n}\left(M\left(\frac{\left|\Delta^{k-1} a_{i j}-L\right|}{\rho}+\frac{\left|\Delta^{k-1} a_{i+1, j}-L\right|}{\rho}+\frac{\left|\Delta^{k-1} a_{i, j+1}-L\right|}{\rho}+\frac{\left|\Delta^{k-1} a_{i+1, j+1}-L\right|}{\rho}\right)\right)^{p_{i j}} \\
\leq D^{2} \cdot \lim _{m, n} \frac{1}{m n} \sum_{i=1}^{m} \sum_{j=1}^{n}\left\{\left(M\left(\frac{\left|\Delta^{k-1} a_{i j}-L\right|}{\rho}\right)\right)^{p_{i j}}+\left(M\left(\frac{\left|\Delta^{k-1} a_{i+1, j}-L\right|}{\rho}\right)\right)^{p_{i j}}\right. \\
\left.+\left(M\left(\frac{\left|\Delta^{k-1} a_{i, j+1}-L\right|}{\rho}\right)\right)^{p_{i j}}+\left(M\left(\frac{\left|\Delta^{k-1} a_{i+1, j+1}-L\right|}{\rho}\right)\right)^{p_{i j}}\right\} . \\
\leq D^{2} \cdot \lim _{m, n} \frac{1}{m n} \sum_{i=1}^{m} \sum_{j=1}^{n}\left\{\left(M\left(\frac{\left|\Delta^{k-1} a_{i j}-L\right|}{\rho}\right)\right)^{p_{i j}}+\left(M\left(\frac{\left|\Delta^{k-1} a_{i+1, j}-L\right|}{\rho}\right)\right)^{p_{i+1, j}}\right. \\
\left.+\left(M\left(\frac{\left|\Delta^{k-1} a_{i, j+1}-L\right|}{\rho}\right)\right)^{p_{i, j+1}}+\left(M\left(\frac{\left|\Delta^{k-1} a_{i+1, j+1}-L\right|}{\rho}\right)\right)^{p_{i+1, j+1}}\right\}
\end{gathered}
$$

$=0$.

Thus $<a_{i j}>\in{ }_{2} W_{0}\left(M, \Delta^{k}, p\right)$ and hence ${ }_{2} W(M, p) \subseteq{ }_{2} W_{0}\left(M, \Delta^{k}, p\right)$.

The inclusion is strict follows from the following example.

Theorem 3.4. (i) If $0<\inf p_{i j} \leq p_{i j}<1$, then ${ }_{2} W\left(M, \Delta^{k}, p\right) \subseteq{ }_{2} W\left(M, \Delta^{k}\right)$.

$$
\text { (ii) If } 1 \leq p_{i j}<\sup p_{i j}<\infty \text {, then }{ }_{2} W\left(M, \Delta^{k}\right) \subseteq{ }_{2} W\left(M, \Delta^{k}, p\right) \text {. }
$$

Proof. The first part of the result follows from the inequality

$$
\frac{1}{m n} \sum_{i=1}^{m} \sum_{j=1}^{n}\left(M\left(\frac{\left|\Delta^{k} a_{i j}-L\right|}{\rho}\right)\right) \leq \frac{1}{m n} \sum_{i=1}^{m} \sum_{j=1}^{n}\left(M\left(\frac{\left|\Delta^{k} a_{i j}-L\right|}{\rho}\right)\right)^{p_{i j}}
$$

and the second part of the result follows from the inequality

$$
\frac{1}{m n} \sum_{i=1}^{m} \sum_{j=1}^{n}\left(M\left(\frac{\left|\Delta^{k} a_{i j}-L\right|}{\rho}\right)\right)^{p_{i j}} \leq \frac{1}{m n} \sum_{i=1}^{m} \sum_{j=1}^{n}\left(M\left(\frac{\left|\Delta^{k} a_{i j}-L\right|}{\rho}\right)\right)
$$

Theorem 3.5. Let $M_{1}$ and $M_{2}$ be two Orlicz functions. Then

$$
{ }_{2} W\left(M_{1}, \Delta^{k}, p\right) \cap{ }_{2} W\left(M_{2}, \Delta^{k}, q\right) \subseteq{ }_{2} W\left(M_{1}+M_{2}, \Delta^{k}, q\right) .
$$

Proof. Let $<a_{i j}>\in{ }_{2} W\left(M_{1}, \Delta^{k}, p\right) \cap{ }_{2} W\left(M_{2}, \Delta^{k}, q\right)$. Then 


$$
\begin{aligned}
& \lim _{m, n} \frac{1}{m n} \sum_{i=1}^{m} \sum_{j=1}^{n}\left(M_{1}\left(\frac{\left|\Delta^{k} a_{i j}-L\right|}{\rho_{1}}\right)\right)^{p_{i j}}=0, \text { for some } \rho_{1}>0 . \\
& \lim _{m, n} \frac{1}{m n} \sum_{i=1}^{m} \sum_{j=1}^{n}\left(M_{2}\left(\frac{\left|\Delta^{k} a_{i j}-L\right|}{\rho_{2}}\right)\right)^{p_{i j}}=0 \text {, for some } \rho_{2}>0 .
\end{aligned}
$$

Let $\rho=\max \left\{\rho_{1}, \rho_{2}\right\}$. The result follows from the following inequality.

$$
\begin{aligned}
& \sum_{i=1}^{m} \sum_{j=1}^{n}\left(\left(M_{1}+M_{2}\right)\left(\frac{\left|\Delta^{k} a_{i j}-L\right|}{\rho}\right)\right)^{p_{i j}} \leq \\
& D\left\{\sum_{i=1}^{m} \sum_{j=1}^{n}\left(M_{1}\left(\frac{\left|\Delta^{k} a_{i j}-L\right|}{\rho_{1}}\right)\right)^{p_{i j}}+\sum_{i=1}^{m} \sum_{j=1}^{n}\left(M_{2}\left(\frac{\left|\Delta^{k} a_{i j}-L\right|}{\rho_{2}}\right)\right)^{p_{i j}}\right\} .
\end{aligned}
$$

Theorem 3.6. The sequence space ${ }_{2} W_{\infty}\left(M, \Delta^{m}, p\right)$ is solid and hence monotone.

Proof. Let $\left\langle a_{i j}>\in{ }_{2} W_{\infty}\left(M, \Delta^{k}, p\right)\right.$ and $\left\langle\alpha_{i j}>\right.$ be a scalar sequence such that $\left|\alpha_{i j}\right| \leq 1$ for all $i, j \in N$.

$$
\begin{aligned}
& \text { Now } M\left(\frac{\left|\alpha_{i j} \Delta^{k} a_{i j}\right|}{\rho}\right) \leq M\left(\frac{\left|\Delta^{k} a_{i j}\right|}{\rho}\right) \\
& \Rightarrow\left(M\left(\frac{\left|\alpha_{i j} \Delta^{k} a_{i j}\right|}{\rho}\right)\right)^{p_{i j}} \leq\left(M\left(\frac{\left|\Delta^{k} a_{i j}\right|}{\rho}\right)\right)^{p_{i j}} \\
& \Rightarrow \sup _{m, n} \frac{1}{m n} \sum_{i=1}^{m} \sum_{j=1}^{n}\left(M\left(\frac{\left|\alpha_{i j} \Delta^{k} a_{i j}\right|}{\rho}\right)\right)^{p_{i j}} \leq \sup _{m, n} \frac{1}{m n} \sum_{i=1}^{m} \sum_{j=1}^{n}\left(M\left(\frac{\left|\Delta^{k} a_{i j}\right|}{\rho}\right)\right)^{p_{i j}}<\infty .
\end{aligned}
$$

Result 3.7. The sequence spaces ${ }_{2} W\left(M, \Delta^{k}, p\right)$ and ${ }_{2} W_{0}\left(M, \Delta^{k}, p\right)$ are not monotone and hence are not solid.

Proof. The result follows from the following example.

Example 3.1. Let $M(x)=x^{p}, p \geq 1$. Then the double sequence $<a_{i j}>$ defined by $a_{i j}=1$ for all $i, j \in N$ belongs to ${ }_{2} W\left(M, \Delta^{k}, p\right)$ and ${ }_{2} W_{0}\left(M, \Delta^{k}, p\right)$. Consider its pre-image $<b_{i j}>$ defined as

$$
b_{i j}=\left\{\begin{array}{l}
a_{i j}, \text { if } i+j \text { is odd. } \\
0, \text { otherwise. }
\end{array}\right.
$$

Then $\left\langle b_{i j}>\right.$ belongs neither to ${ }_{2} W\left(M, \Delta^{k}, p\right)$ nor to ${ }_{2} W_{0}\left(M, \Delta^{k}, p\right)$ for any $k$. Hence the spaces ${ }_{2} W\left(M, \Delta^{k}, p\right)$ and ${ }_{2} W_{0}\left(M, \Delta^{k}, p\right)$ are not monotone and by Remark 3 these are not solid also. symmetric.

Result 3.8. The sequence spaces ${ }_{2} W\left(M, \Delta^{k}, p\right),{ }_{2} W_{0}\left(M, \Delta^{k}, p\right)$ and ${ }_{2} W_{\infty}\left(M, \Delta^{k}, p\right)$ are not

Proof. To prove the results consider the following examples. 
Example 3.2. Let $M(x)=x^{2}, k=2$. Consider the sequence $<a_{i j}>$ defined by

$$
a_{i j}=\left\{\begin{array}{c}
1, \text { if } i \text { is odd for all } j \in N . \\
-1, \text { otherwise. }
\end{array}\right.
$$

Then $\Delta^{2} a_{i j}=0$ for all $i, j \in N$.

Let $<b_{i j}>$ be a rearrangement of the sequence $<a_{i j}>$ defined by

$$
b_{i j}=\left\{\begin{array}{c}
-1, \text { if } i+j \text { is even. } \\
1, \text { otherwise. }
\end{array}\right.
$$

Then $\quad \Delta^{2} b_{i j}=\left\{\begin{array}{l}-16, \text { if } i+j \text { is even. } \\ 16, \text { otherwise. }\end{array}\right.$

Here $<a_{i j}>\in{ }_{2} W_{0}\left(M, \Delta^{k}, p\right) \subseteq{ }_{2} W\left(M, \Delta^{k}, p\right)$ but $<b_{i j}>\notin{ }_{2} W\left(M, \Delta^{k}, p\right)$.

Example 3.3. Let $M(x)=x^{p}, p \geq 1, k=2, p_{i j}=2$ for all $i, j \in N$. Consider the sequence $<a_{i j}>$ defined by

$$
a_{i j}=\left\{\begin{array}{l}
0, \text { if } i \text { is even for all } j \in N . \\
i, \text { otherwise. }
\end{array}\right.
$$

Then $\Delta a_{i j}=0$ for all $i, j \in N$.

Let $<b_{i j}>$ be a rearrangement of the sequence $<a_{i j}>$ defined by

$$
b_{i j}=\left\{\begin{array}{l}
0, \text { if } i+j \text { is odd. } \\
i, \text { otherwise }
\end{array}\right.
$$

Then $\quad \Delta^{2} b_{i j}=\left\{\begin{array}{l}8 i+8, \text { if } i+j \text { is even. } \\ -8 i-8, \text { otherwise. }\end{array}\right.$

Here $<a_{i j}>\in{ }_{2} W_{\infty}\left(M, \Delta^{k}, p\right)$ but $<b_{i j}>\notin{ }_{2} W_{\infty}\left(M, \Delta^{k}, p\right)$. 


\section{REFERENCES}

1. Basarir, M. and Sonalcan, O.: On some double sequence spaces; J. Indian Acad. Math. 21(2), (1999); 193-200.

2. Bromwich T.J.IA: An Introduction to the Theory of Infinite Series; MacMillan and Co. Ltd. New york (1965).

3. Esi, A.: Some new sequence spaces defined by Orlicz functions; Bull. Inst. Math. Acad. Sinica.; 27(1) (1999), 71-76.

4. Esi, A. and Et, M.: Some new sequence spaces defined by a sequence of Orlicz functions; Indian J. Pure. Appl. Math.; 31(8) (2000), 967-972.

5. Et, M.: On some new Orlicz sequence spaces; J. Analysis; 9 (2001), 21-28.

6. Hardy G.H.: On the convergence of certain multiple series; Proc. Camb. Phil. Soc.; 19 (1917).

7. Kamthan, P.K. and Gupta, M.: Sequence Spaces and Series: Marcel Dekker, 1980.

8. Kizmaz, H: On certain sequence spaces; Canad. Math. Bull., 24 (1981), 169 -176.

9. Krasnoselkii, M.A. and Rutitsky, Y.B.: Convex function and Orlicz Spaces; Groningen Netherlands, 1961.

10. Lindenstrauss, J. and Tzafriri, L.: On Orlicz sequence spaces: Israel J. Math. 10 (1971), 379-390.

11. Maddox, I.J.: Spaces of strongly summable sequences. Quart. Jour. Math. (Oxford $2^{\text {nd }}$ Ser), vol. 18, no.72(1976), 345-355.

12. Moricz, F: Extension of the spaces $c$ and $c_{0}$ from single to double sequences; Acta. Math. Hungerica.; 57(1-2), (1991), 129 -136.

13. Moricz, F. and Rhoades B.E.: Almost convergence of double sequences and strong regularity of summability matrices; Math. Proc. Camb. Phil. Soc.; 104 (1988), 283-294.

14. Nakano H.: Modular sequence spaces; Proc. Japan Acad.; 27 (1951), 508 - 512.

15. Simons, S.: The sequence spaces $l\left(p_{v}\right)$ and $m\left(p_{v}\right)$. Proc. London Math. Soc.,(3) 15 (1965), 422-436.

16. Tripathy B.C.: Generalized difference paranormed statistically convergent sequences defined by Orlicz function in a locally convex spaces; Soochow J. Math. 30(4)(2004), 431446.

17. Tripathy B.C. and Sarma B.: Statistically convergent double sequence spaces defined by Orlicz functions; Soochow J. Math.; 32(2)(2006), 211-221.

18. Tripathy B.C., Choudhury B. and Sarma B.: Some Difference Double Sequence Spaces Defined By Orlicz Function, Kyungpook Math. J. 48(2008), 613-622.

19. Zygumd, A.: Trigonometric Series, vol II , Cambridge (1993) . 